\title{
Packing fraction of crystalline structures of binary hard spheres: A general equation and application to amorphization
}

\author{
H. J. H. Brouwers \\ Faculty of Engineering Technology, University of Twente, P.O. Box 217, 7500 AE Enschede, The Netherlands
}

(Received 21 November 2007; revised manuscript received 21 January 2008; published 15 July 2008)

\begin{abstract}
In a previous paper analytical equations were derived for the packing fraction of crystalline structures consisting of bimodal randomly placed hard spheres [H. J. H. Brouwers, Phys. Rev. E 76, 041304 (2007)]. The bimodal packing fraction was derived for the three crystalline cubic systems: viz., face-centered cubic, bodycentered cubic, and simple cubic. These three equations appeared also to be applicable to all 14 Bravais lattices. Here it is demonstrated, accounting for the number of distorted bonds in the building blocks and using graph theory, that one general packing equation can be derived, valid again for all lattices. This expression is validated and applied to the process of amorphization.
\end{abstract}

DOI: 10.1103/PhysRevE.78.011303

PACS number(s): 45.70. $-\mathrm{n}, 71.55 . \mathrm{Jv}$

\section{MEAN SPHERE VOLUME}

For a stacking of equal spheres in a cubic structure, the packing fraction follows from the number of spheres, $N$, with diameter $d$ in the unit cell, sphere volume $\Omega$, and unit cell volume $V_{\text {cell }}$ :

$$
f_{1}=\frac{N \Omega}{V_{\text {cell }}}=\frac{N \frac{\pi}{6} d^{3}}{\ell^{3}},
$$

with $\ell$ as lattice constant or lattice parameter [1]. For the face-centered cubic (fcc) structure, $N=4$ and $\ell=2^{1 / 2} d$; for the body-centered cubic (bcc) structure, $N=2$ and $\ell=2 d / 3^{1 / 2}$; and for the simple cubic (sc) structure, $N=1$ and $\ell=d$, yielding the monosized packing fractions $f_{1}^{\text {fcc }}=2^{1 / 2} \pi / 6, f_{1}^{\text {bcc }}$ $=3^{1 / 2} \pi / 8$, and $f_{1}^{\mathrm{sc}}=\pi / 6$, respectively. For an arrangement of bimodal spheres, the mean sphere volume readily follows as

$$
\Omega=X_{L} \Omega_{L}+\left(1-X_{L}\right) \Omega_{S}=\frac{\pi\left[X_{L} d_{L}^{3}+\left(1-X_{L}\right) d_{S}^{3}\right]}{6} .
$$

$X$ is the mole fraction, and the subscripts $S$ and $L$ refer to small and large spheres, respectively. The magnitude of the bimodal cell volume is addressed in the next section.

\section{CELL VOLUME}

In [1], using the statistically probable combinations of small and large spheres, the cell volume followed as

$$
\begin{aligned}
V_{\text {cell }}= & \sum_{i=0}^{n}\left\{\left(\begin{array}{c}
n \\
i
\end{array}\right) X_{L}^{n-i}\left(1-X_{L}\right)^{i}\left[\frac{n-i}{n} \ell_{L}^{3}+\frac{i}{n} \ell_{S}^{3}+\lambda\left(\ell_{L}^{3}-\ell_{S}^{3}\right)\right]\right\} \\
& -\lambda\left[X_{L}^{n}+\left(1-X_{L}\right)^{n}\right]\left(\ell_{L}^{3}-\ell_{S}^{3}\right),
\end{aligned}
$$

with $n$ the number of spheres that form the elementary building blocks of the crystal structure considered.

In Eq. (3), the lattice distortion is accounted for by the factor $\lambda$, which allows for the spacing resulting from the combination of the large and small spheres in the cells in which they both appear. The exact mathematical nature of the volume mismatch is not known; here, a linear dependence as a first-order perturbation is taken, such that the distortion term indeed tends to zero when $\ell_{S}$ tends to $\ell_{L}$-that is, when a monosized system is obtained and $V_{\text {cell }}$ should tend to $\ell_{S}^{3}=\ell_{L}^{3}[1]$. The two last terms on the righthand side provide that the building blocks consisting of identical spheres, large or small only, are counted as nondistorted (i.e., in the state of close monosized packing). So Eq. (3) accounts for nondistortion in the case of $i=0$ and $i=n$. For all other values of $i$-i.e., concerning combinations of unequal spheres-Eq. (3) implicitly assumes that the distortion term $\lambda$ is identical for all $i$. Alternatively, in this article the ansatz is made that the distortion is proportional to the number of distorted contacts in an elementary building block, which will depend on $i$ (the composition). This approach was also followed in [2] for describing the binary random packing of disks in two dimensions. The model presented in this article is based on a statistical approach of the occurrence of even and odd bonds in a bimodal structure. This is a refinement of [1], in which only distinction was made between building blocks consisting of monosized spheres on the one hand and mixed building blocks on the other. The different latter ones are now treated differently as well, being self-consistent with the distinction already made in [1], and it is conjectured that this is a more realistic representation of distorted building blocks (i.e., building blocks containing nonidentical spheres).

As a first step, in Fig. 1(a), for example, a twodimensional (2D) Platonic graph of the tetrahedron is depicted, the four spheres touching together being the elementary building blocks of the fcc structure [1,3], the spheres represented by vertices and their contacts by edges. This graph is planar as it can be drawn so that no edges cross. Since every vertex has the same number of edges-i.e., they are all from the same degree $\xi$ - the graph is regular. In addition, this fcc graph is a so-called complete graph as every pair of distinct vertices is adjacent as well. In general, the total number of edges (here contacts), $b_{t}$, of a regular graph of degree (or valence) $\xi$ with $n$ vertices reads 


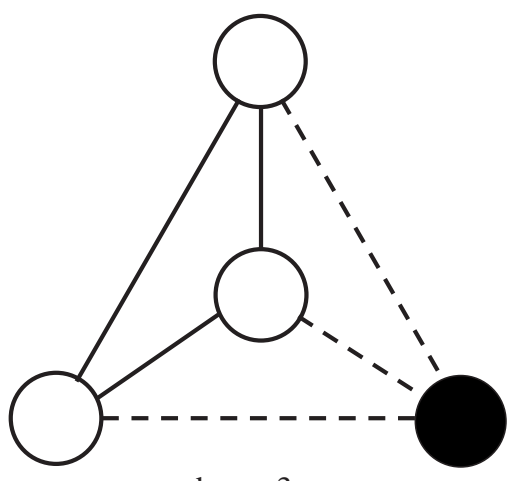

(a)

$\mathrm{b}_{\mathrm{LS}}=3$

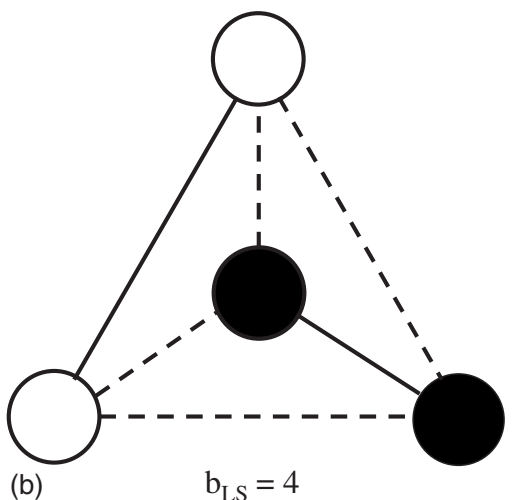

FIG. 1. Alternating Platonic graphs of the tetrahedron ( $n$ $=4, \quad \xi=3$ ). (a) One odd vertex. (b) Two odd vertices.

$$
b_{t}=\frac{1}{2} n \xi \quad(2 \leq \xi \leq n-1) .
$$

For $\xi=2$, a simple circuit between $n$ vertices is obtained and $\xi=n-1$ corresponds to the complete graph. Now, accounting for the number of distorted contacts the cell volume can be formulated as

$$
\begin{aligned}
V_{\text {cell }}= & \sum_{i=0}^{n}\left[\left(\begin{array}{l}
n \\
i
\end{array}\right) X_{L}^{n-i}\left(1-X_{L}\right)^{i}\left(\frac{n-i}{n} \ell_{L}^{3}+\frac{i}{n} \ell_{S}^{3}\right)\right. \\
& \left.+\psi_{i} X_{L}^{n-i}\left(1-X_{L}\right)^{i}\left(\ell_{L}^{3}-\ell_{S}^{3}\right)\right],
\end{aligned}
$$

for which now the distortion parameter is proportional to number of distorted contacts $\left(b_{L S}\right)$ - that is to say, the number of edges (or contacts) between uneven pairs of vertices (or spheres),

$$
\psi_{i}=\left(\begin{array}{l}
n \\
i
\end{array}\right) \lambda_{i}=C\left(\begin{array}{l}
n \\
i
\end{array}\right) \frac{b_{L S}(i)}{2 b_{t}},
$$

in which $C$ is a proportionality constant. In contrast to $\lambda$ appearing in Eq. (3), the distortion terms $\psi_{i}$ and $\lambda_{i}$ are functions of the number of odd vertices $i$ in the building block.

For the fcc structure-that is to say, $n=4$ and $\xi=n-1$, with Eq. (4) $-b_{t}=6$ is readily obtained; see also Fig. 1(a). For $i=0$ and $i=4$ there are no distorted contacts, so the number of distorted contacts $b_{L S}(i=0)=b_{L S}(i=4)=0$ and $\psi=\lambda$ $=0$, as already accounted for by Eq. (3) and in [1]. For $i=1$ (or 3), on the other hand, $b_{L S}(i=1)=b_{L S}(i=3)=3$; see Fig. 1(a), in which one vertex (sphere) alternates from the three others and in which the distorted edges are indicated by dashed lines (as in all figures). For $i=2$, Fig. 1(b) shows, irrespective the vertex occupation by the second odd sphere, $b_{L S}(i=2)=4$. Summarizing, considering Eq. (6), it follows that

$$
\begin{gathered}
\psi_{0}=\psi_{4}=0, \\
\psi_{1}=\psi_{3}=C, \\
\psi_{2}=2 C .
\end{gathered}
$$

Note that Eq. (3) also accounted for Eq. (7)-i.e., the cases of no mismatch $(i=0$ and $i=4)$ - but that the distortion parameters of all other $i$ now account for the number of distorted contacts. Inserting Eqs. (7)-(9) into Eq. (5), using $n$ $=4$, yields

$$
V_{\text {cell }}=X_{L} \ell_{L}^{3}+\left(1-X_{L}\right) \ell_{S}^{3}+C X_{L}\left(1-X_{L}\right)\left(\ell_{L}^{3}-\ell_{S}^{3}\right) .
$$

To obtain the two first terms on the right-hand side (so-called Retger's equation [1]), an equation has been used that governs the expected value of the probability mass function of the binomial distribution:

$$
\sum_{i=0}^{n}\left[\left(\begin{array}{l}
n \\
i
\end{array}\right) i X_{L}^{n-i}\left(1-X_{L}\right)^{i}\right]=n\left(1-X_{L}\right) .
$$

Here, as in $[1,4,5]$, it is assumed that upon the introduction of small spheres in a structure of large spheres only, it will not change the cell volume; in other words, each small sphere will be able to rattle in its cage formed by the larger sphere volume. Mathematically, this implies that the first derivative of the cell volume with respect to $X_{L}$ at the large sphere side $\left(X_{L}=1\right)$ equals zero- that is to say,

$$
\left.\frac{d V_{\text {cell }}}{d X_{L}}\right|_{X_{L}=1}=0,
$$

Eq. (10) yielding $C=1$, and hence

$$
V_{\text {cell }}=\ell^{3}=X_{L} \ell_{L}^{3}+\left(1-X_{L}\right) \ell_{S}^{3}+X_{L}\left(1-X_{L}\right)\left(\ell_{L}^{3}-\ell_{S}^{3}\right) .
$$

This equation is very similar to the fcc cell volume derived in [1]:

$$
\begin{aligned}
V_{\text {cell }}= & X_{L} \ell_{L}^{3}+\left(1-X_{L}\right) \ell_{S}^{3}+X_{L}\left(1-X_{L}\right)\left(1-\frac{1}{2} X_{L}\left(1-X_{L}\right)\right) \\
& \times\left(\ell_{L}^{3}-\ell_{S}^{3}\right) .
\end{aligned}
$$

In [1] the packing fraction based on both equations has been compared [Fig. 4(a) there] and a slight difference could be observed. Furthermore, in [1], Eq. (14) was also compared with empirical data from Luck et al. [6] and good agreement was seen. In Table I, both Eqs. (13) and (14) are included (using $\ell=V_{\text {cell }}^{1 / 3}$ ), as well as the bimodal lattice parameters following from Retger's and Vegard's equations [1]. One can see that the difference between Eqs. (13) and (14) is very small and only noticeable when the composition is close to 
TABLE I. Lattice parameters measured by Luck et al. [6] and computed values by employing Eq. (13), Eq. (14), and Retger's and Vegard's equations [1].

\begin{tabular}{lccccccccc}
\hline \hline & $\begin{array}{c}\ell_{L} \\
c_{L}\end{array}$ & $\begin{array}{c}\ell_{S} \\
(\mathrm{~nm})\end{array}$ & $u$ & $X_{L}$ & $\begin{array}{c}\text { Measured } \\
(\mathrm{nm})\end{array}$ & $\begin{array}{c}\text { Equation (13) } \\
(\mathrm{nm})\end{array}$ & $\begin{array}{c}\text { Equation (14) } \\
(\mathrm{nm})\end{array}$ & $\begin{array}{c}\ell_{\text {Retgers }} \\
(\mathrm{nm})\end{array}$ & $\begin{array}{c}\ell_{\text {Vegard }} \\
(\mathrm{nm})\end{array}$ \\
\hline 0.500 & 414 & 375 & 1.104 & 0.426 & 403 & 402 & 401 & 393 & 392 \\
0.901 & 472 & 375 & 1.259 & 0.048 & 385 & 386 & 386 & 381 & 380 \\
\hline \hline
\end{tabular}

parity (i.e., $X_{L}$ approaches 0.5 ), in which case Eq. (13) comes even closer to the measured value (Table I). When metal or metalloids are alloyed, their measured lattice parameter is the result of geometrical dilatation [Eq. (13)] and other effects (e.g., valence, electronegativity [8]), which can now be distinguished.

Next, for the bcc lattice, the smallest building block is formed by the four spheres that enclose the tetrahedral interstice, each sphere touching two other ones. Figures 2(a) and 2(b) represent 2D graphs of systems with one and two odd vertices, respectively. The bcc graph can therefore be seen as a circuit consisting of again four $(n)$ vertices of degree $2(\xi)$, yielding in total four edges $\left[b_{t}\right.$; see Eq. (4)].

Again, for $i=0$ and $i=4$ there are no distorted contacts, so $b_{L S}(i=0)=b_{L S}(i=4)=0$ and $\psi=\lambda=0$. From Fig. 2(a) it can be seen that for $i=1$ (and $i=3), b_{L S}(i=1)=b_{L S}(i=3)=2$. For $i$ $=2$, there is a $2 / 3$ probability that $b_{L S}(i=2)=2$ and a $1 / 3$ probability that $b_{L S}(i=2)=4$ [Fig. 2(b)], yielding as mathematical expectation $b_{L S}(i=2)=8 / 3$. Substituting aforesaid values into Eq. (6) again yields Eqs. (7)-(10), with Eqs. (5) and (11); this results in Eq. (10), and with Eq. (12), again Eq. (13) is again obtained. This result reveals that the cellular volumes of the fcc and bcc structures are affected in the same way upon combining two sphere sizes, though the absolute magnitudes of the lattice parameter $\ell$ and cell volume $V_{\text {cell }}$ differ.

A similar analysis as for the fcc and bec structures can be performed for the sc structure, having a building block that consist of eight $(n)$ spheres that each have three $(\xi)$ contacts, yielding total contacts $b_{t}=12$ [Eq. (4)]. For $i=0$ and $i=8$ there are no distorted contacts, so $b_{L S}(i=0)=b_{L S}(i=8)=0$ and $\psi=\lambda=0$. For $i=1$ (or $i=7$ ) in Fig. 3(a) the Platonic graph of this structure is given [3], which reveals that $b_{L S}(i=1)$ $=b_{L S}(i=7)=3$. In Fig. 3(b) the probabilities of the configurations with two odd vertices are given, yielding $b_{L S}(i=2)$ $=b_{L S}(i=6)=36 / 7$. In Fig. 3(c) the different configurations and their probabilities pertaining to $i=3$ (or $i=5$ ) are presented, yielding the expectation $b_{L S}(i=3)=b_{L S}(i=5)=45 / 7$. Finally, the possible configurations belonging to $i=4$ are given in Fig. 3(d) - i.e., when half of the vertices are odd- having an expected value $b_{L S}(i=4)=48 / 7$. Inserting these $b_{L S}(i)$ values in Eq. (6) yields

$$
\begin{gathered}
\psi_{0}=\psi_{8}=0, \\
\psi_{1}=\psi_{7}=C, \\
\psi_{2}=\psi_{6}=6 C, \\
\psi_{3}=\psi_{5}=15 C, \\
\psi_{4}=20 C .
\end{gathered}
$$

Substituting Eqs. (15)-(19) into Eq. (5) with $n=8$, applying Eq. (11), again yields Eq. (10). Subsequently, using Eq. (12), again yields Eq. (13). Hence, the analysis of the bimodal sc structure, taking account of the number of distorted vertices and their probability, yields the same equation as obtained for the fcc and bcc structures.

\section{PACKING FRACTION}

Combining the bimodal sphere volume, Eq. (2), with the bimodal cell volume, Eq. (13), and using the size ratio

$$
u=\frac{\ell_{L}}{\ell_{S}}=\frac{d_{L}}{d_{S}}
$$

yields one general expression that governs the ratio of bimodal packing fraction $\eta$ and unimodal packing fraction $f_{1}$ :

$$
\frac{\eta}{f_{1}}=\frac{X_{L}\left(u^{3}-1\right)+1}{X_{L}\left(u^{3}-1\right)+1+\left(1-X_{L}\right) X_{L}\left(u^{3}-1\right)},
$$

which holds for all three cubic structures of the cubic crystal system. Equation (21) differs slightly only from the bimodal fcc and bcc packing fraction expressions derived in [1].

From Eq. (21) it follows that the packing fraction near $u=1$ can be described as

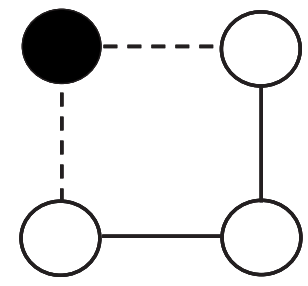

(a) $\mathrm{b}_{\mathrm{LS}}=2$

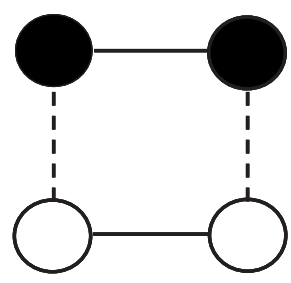

(b) $\mathrm{p}=2 / 3, \mathrm{~b}_{\mathrm{LS}}=2$

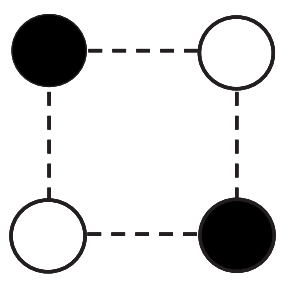

$\mathrm{p}=1 / 3, \mathrm{~b}_{\mathrm{LS}}=4$
FIG. 2. Alternating graphs of the bcc structure $(n=4, \xi=2)$. (a) One odd vertex. (b) Two odd vertices and the probability of the number distorted edges. 


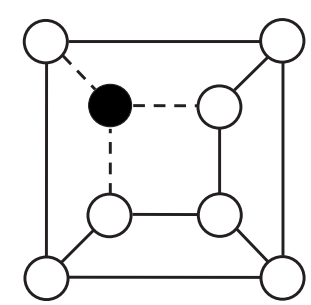

(a) $b_{\mathrm{LS}}=3$

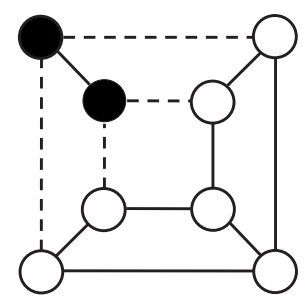

(b) $\mathrm{p}=3 / 7, \mathrm{~b}_{\mathrm{LS}}=4$

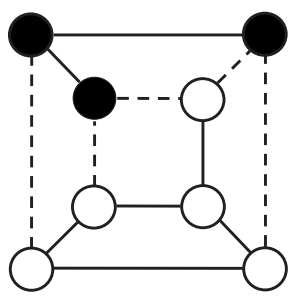

(c) $\mathrm{p}=3 / 7, \mathrm{~b}_{\mathrm{LS}}=5$

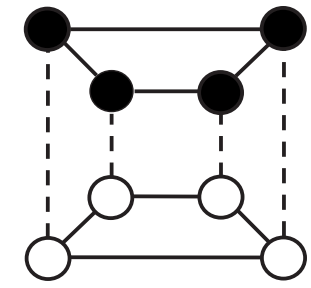

(d) $\mathrm{p}=4 / 35, \mathrm{~b}_{\mathrm{LS}}=4$

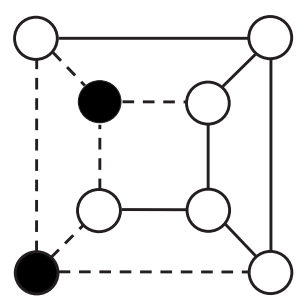

$\mathrm{p}=4 / 7, \mathrm{~b}_{\mathrm{LS}}=6$

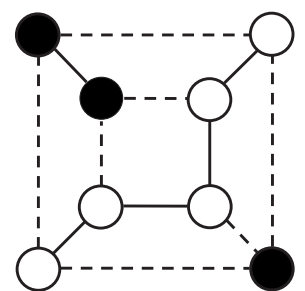

$\mathrm{p}=3 / 7, \mathrm{~b}_{\mathrm{LS}}=7$

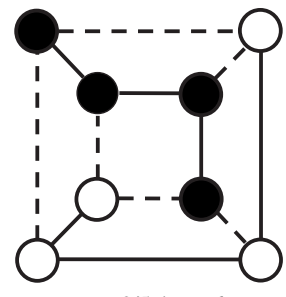

$p=2 / 5, b_{L S}=6$

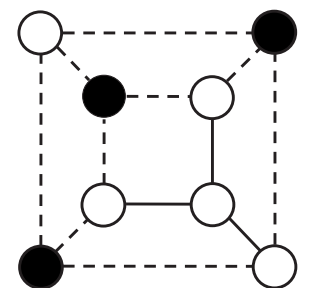

$\mathrm{p}=1 / 7, \mathrm{~b}_{\mathrm{LS}}=9$

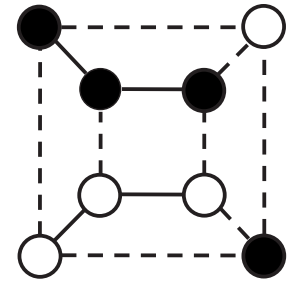

$\mathrm{p}=16 / 35, \mathrm{~b}_{\mathrm{LS}}=8$

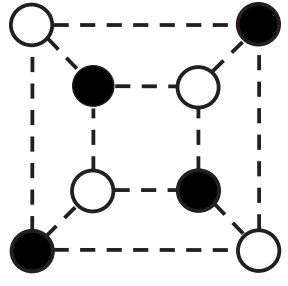

$\mathrm{p}=1 / 35, \mathrm{~b}_{\mathrm{LS}}=12$
FIG. 3. Alternating Platonic graphs of the cube $(n=8, \quad \xi=3)$. (a) One odd vertex. (b) Two odd vertices and the probability of the number distorted edges. (c) Three odd vertices and the probability of the number distorted edges. (d) Four odd vertices and the probability of the number distorted edges.

$$
\begin{aligned}
\eta\left(u, X_{L}\right) & =f_{1}+f_{1}\left(1-X_{L}\right) X_{L}\left(u^{3}-1\right) \\
& =f_{1}+3 f_{1}\left(1-X_{L}\right) X_{L}(u-1) .
\end{aligned}
$$

Furthermore, Eqs. (21) and (22) yield as gradient $\beta=$ $-3 / 4\left(1-f_{1}\right)$ for all cubic structures $[1,7]$.

In [1] it was reasoned that the three bimodal cubic lattices also stand for all other 11 Bravais lattices of the 5 other crystal systems: triclinic, monoclinic, orthorhombic, hexagonal-rhombohedral, and tetragonal. As their buildings blocks and number of bonds correspond to the cubic lattices, here it can be concluded again that Eqs. (21) and (22) are also applicable to all 14 Bravais lattices.

\section{AMORPHIZATION}

In [1] the packing fraction of random close packing (rcp), taken from [7], and the bimodal crystalline packing fraction of fcc and bcc were combined and the mode of densest packing, glass-amorphous or crystalline, determined. Using the bimodal packing fractions derived here, Eq. (21), and Eq. (1) from [1], the glass-fcc crossover line now reads

$$
\begin{aligned}
f_{1}^{\mathrm{rcp}} & +4 \beta^{\mathrm{rcp}} f_{1}^{\mathrm{rcp}}\left(1-f_{1}^{\mathrm{rcp}}\right)\left(1-X_{L}\right) X_{L}\left(\frac{(z+1)^{1 / 3}-z^{1 / 3}}{z^{1 / 3}}\right) \\
& =f_{1}^{\mathrm{fcc}}\left(\frac{z+X_{L}}{z+X_{L}+1+X_{L}\left(1-X_{L}\right)}\right)
\end{aligned}
$$

in which is introduced

$$
z=\frac{\Omega_{S}}{\Omega_{L}-\Omega_{S}}=\frac{1}{u^{3}-1}, \quad u-1=\frac{(z+1)^{1 / 3}-z^{1 / 3}}{z^{1 / 3}} .
$$

For $u$ close to unity, the approximate equation (22) is equated with Eq. (1) from [1], yielding the explicit equation

$$
\frac{1}{u-1}=\left(1-X_{L}\right) X_{L}\left(\frac{4 \beta^{\mathrm{rcp}} f_{1}^{\mathrm{rcp}}\left(1-f_{1}^{\mathrm{rcp}}\right)+3 f_{1}^{\mathrm{fcc}}}{f_{1}^{\mathrm{fcc}}-f_{1}^{\mathrm{rcp}}}\right) \text {. }
$$

The resulting $z$ [using Eq. (24)] versus $X_{L}$ is included in Fig. $4(\mathrm{a})$, using $f_{1}^{\mathrm{fcc}}=0.74, f_{1}^{\mathrm{rcp}}=0.64$, and $\beta^{\mathrm{rcp}}=0.20$ [1]. One can see that this approximate equation matches the full equation reasonably well. The glass-bcc crossover line also follows from Eqs. (23) and (25) when $f_{1}^{\text {fcc }}$ is replaced by $f_{1}^{\text {bcc }}(=0.68)$-i.e., the monosized fcc packing fraction by the 

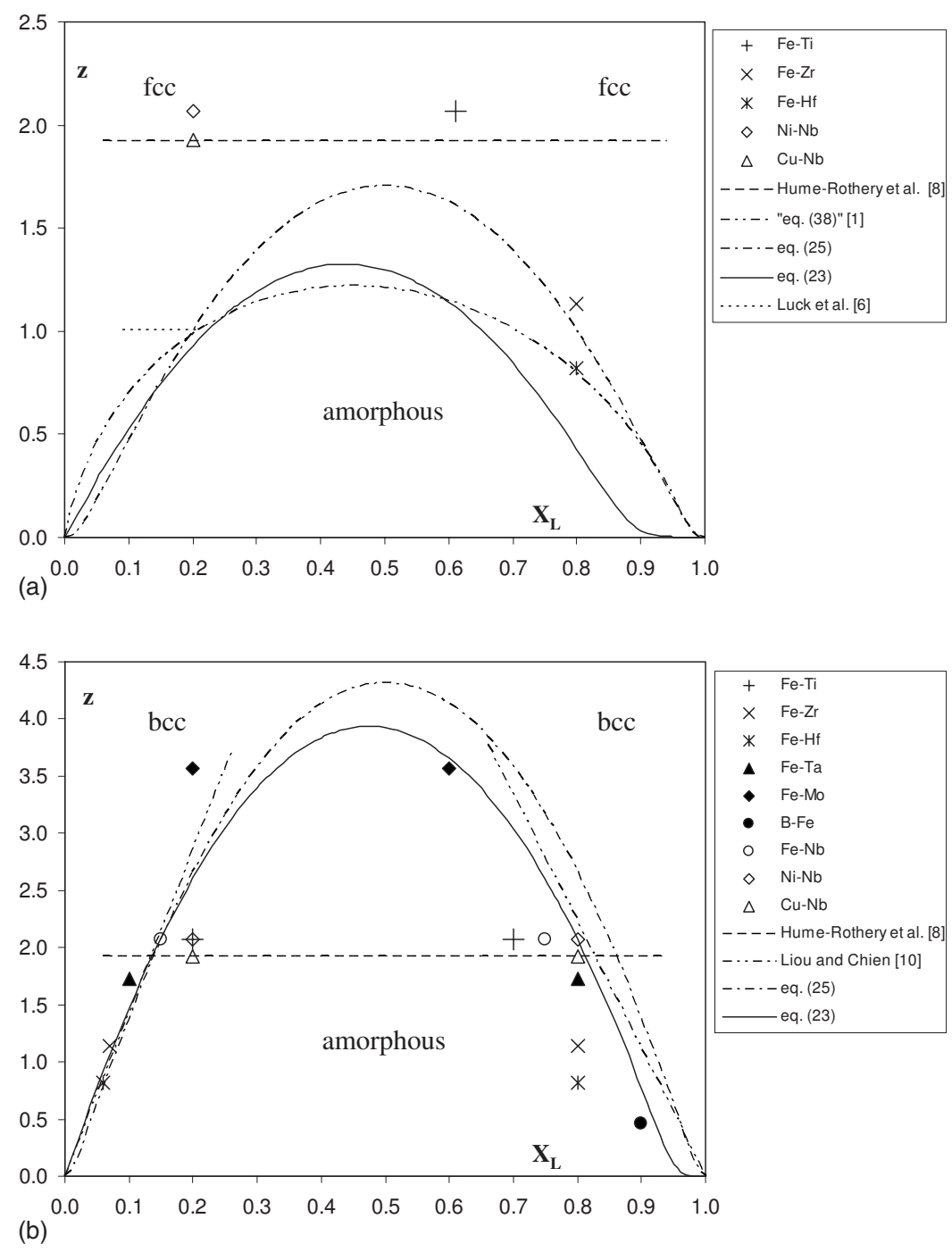

FIG. 4. (a): Diagram of closest bimodal packing using the crossover line (equal packing fraction) of bimodal fcc-hcp and random close packings (rcp). (b): Diagram of closest bimodal packing using the crossover line (equal packing fraction) of bimodal bcc and rcp. monosized bcc packing fraction. In Fig. 4(b) the resulting $z$ versus $X_{L}$ are included.

In [1] the glass-forming ability of metals was analyzed using the hard-sphere systems, and this will also be analyzed with the models derived here. The formation and stability of binary alloys upon quenching has been studied intensively in the past. In an early paper Hume-Rothery et al. [8] suggested that a maximum atomic size ratio of $14 \%-15 \%$ is favorable for the formation of substitutional solid solutions and this "amorphization rule" was linked to alloys with atom concentrations exceeding 5\%. The resulting threshold line $u=1.15$ $(z=1.92)$ with $5 \% \leq X_{L} \leq 95 \%$ is drawn in Figs. 4(a) and 4(b) as well. This general equation does not account for the concentration of the constituents, which plays a role and should not be ignored. In Fig. 4(a) also the fcc stability threshold based on crystallization of suspended colloidal spheres, which are often used for studying phase transitions, is included [Eq. (38) with polydispersity $\sigma=10 \%$ in [1]]. In Fig. 4(a), also the crossover concentration range $9 \%<X_{L}$ $<21 \%$ for a colloidal system with $z=1.006$, as observed by Luck et al. [6] and explained in [1], is drawn. This horizontal line almost touches the threshold line prescribed by Eq. (23) and actually intersects with the approximate threshold given by Eq. (25). Based on this comparison, one can conclude that both thresholds are in excellent agreement with the empirical findings in [6]. In general, the derived threshold appears to be in line with foregoing numerical and experimental findings in regard to amorphization of colloidal systems.

Egami and Waseda [9] and Liou and Chien [10] experimentally investigated the effect of both atomic size ratio and atomic concentration on amorphization ability. For a number of binary metals, Liou and Chien [10] determined the concentration threshold of amorphous or crystalline phase formation by quenching binary alloys, which were summarized in Table III in [1], and their results for the fcc-hcp and bcc hosts are set out in Figs. 4(a) and 4(b), respectively. Some alloys appear in both graphs as in [1], since it appeared that some hcp-fcc hosts actually adopt a bcc structure when alloyed with a bcc solute; i.e., the small and large atom-rich sides then take a bcc structure and Fig. 4(b) prevails. Accordingly, in Fig. 4(b) also the two empirical fits by Liou and Chien [10] are included (see [1]) that govern the threshold concentration versus scaled size ratio. Indeed these line fits are compatible with their experimental findings. Both lines predict the threshold well near both the small and large atomrich compositions.

From the crossover lines determined here, the threshold is continuous in the entire compositional range and has a para- 


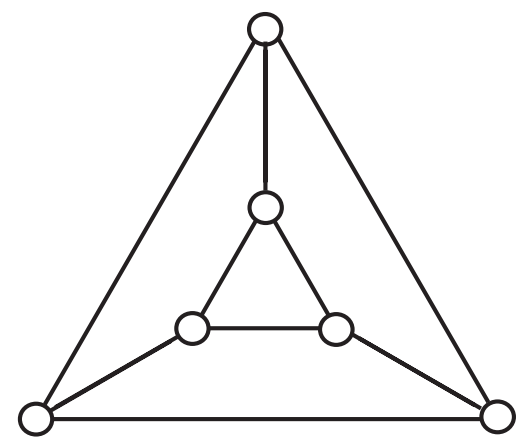

(a)

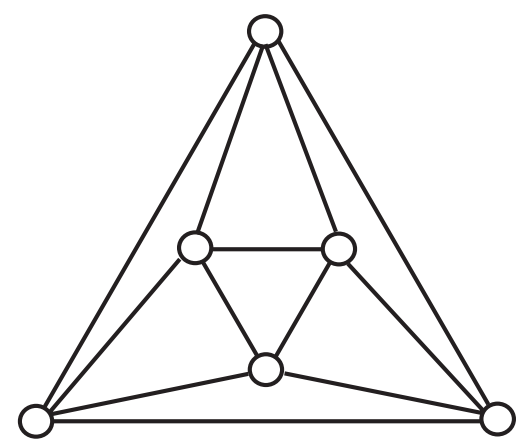

(b)

FIG. 5. (a) Graph representing three hexagonally stacked spheres in a plane, with on top of them the three other spheres, hexagonally packed in a plane as well, each lower sphere touching one top sphere $(n=6, \xi=3)$. (b) Platonic graph of the octahedron $(n=6, \xi=4)$.

bolic shape, and the threshold $z$ and related $u$ depend on the structure (bcc or fcc-hcp). For the bcc structure the maximum crossover $z$ amounts to 3.934 and minimum $u=1.078$, and for the fcc-hcp structure the maximum $z=1.325$ and minimum $u=1.206$ [all based on Eq. (23)].

From Figs. 4(a) and 4(b) it follows that Eq. (23) and the approximate equation (25) predict the empirically observed crossover threshold of the considered colloidal systems and of the alloys remarkably well (and better than the equations presented in [1]), especially when it is realized that the present model is solely based on an analytical analysis, without the introduction of a fitting parameter. In the entire compositional range and for many diameter ratios, Eqs. (23) and (25) signal correctly when the quenched alloy favors crystalline or amorphous phase formation. It should be realized that the obtained crossover line pertains to local concentrations; in the case of phase separations, they may differ from the overall concentration and various amorphous and crystalline regions may occur simultaneously. For instance, from Fig. 4(a) it can be learned that a homogeneous amorphous mix of $z=1$ and overall mole concentration $X_{L}=0.3$ could turn into a two-phase material, one fcc phase having $X_{L}=0.1$ and comprising $1 / 3$ of the spheres (or atoms), and another amorphous phase with $X_{L}=0.4$ and taking the other $2 / 3$ of the spheres (or atoms).

\section{GRAPH GENERALIZATION}

The computation of the possible bimodal configurations for the fcc, bcc, and sc lattices as executed above, resulting in Eqs. (7)-(9) and Eqs. (15)-(19), shows that in general terms $\psi_{i}$ reads

$$
\psi_{i}=C\left(\begin{array}{c}
n-2 \\
i-1
\end{array}\right) \quad(1 \leq i \leq n-1)
$$

so that indeed

$$
\begin{aligned}
& \sum_{i=1}^{n-1} \psi_{i} X_{L}^{n-i}\left(1-X_{L}\right)^{i} \\
& \quad=C \sum_{i=1}^{n-1}\left(\begin{array}{c}
n-2 \\
i-1
\end{array}\right) X_{L}^{n-i}\left(1-X_{L}\right)^{i} \\
& =C X_{L}\left(1-X_{L}\right) \sum_{i=1}^{n-1}\left(\begin{array}{l}
n-2 \\
i-1
\end{array}\right) X_{L}^{n-i-1}\left(1-X_{L}\right)^{i-1} \\
& =C X_{L}\left(1-X_{L}\right),
\end{aligned}
$$

which appears on the right-hand side of Eq. (10) in the cases $n=4(\xi=2$ and $\xi=4)$ and $n=8(\xi=3)$, as examined above.

Equation (27) can be incorporated in the original equation (3) using Eq. (6):

$$
V_{\text {cell }}=\sum_{i=0}^{n}\left[\left(\begin{array}{l}
n \\
i
\end{array}\right) X_{L}^{n-i}\left(1-X_{L}\right)^{i}\left(\frac{n-i}{n} \ell_{L}^{3}+\frac{i}{n} \ell_{S}^{3}+\lambda_{i}\left(\ell_{L}^{3}-\ell_{S}^{3}\right)\right)\right],
$$

with

$$
\lambda_{i}=C \frac{\left(\begin{array}{l}
n-2 \\
i-1
\end{array}\right)}{\left(\begin{array}{l}
n \\
i
\end{array}\right)}=C \frac{i(n-i)}{n(n-1)} \quad(0 \leq i \leq n) .
$$

This equation shows that the distortion term is a quadratic function of the number of odd spheres in the structure, $i$, and symmetrical with respect to $i=n / 2$. This result is based on the present analysis that statistically accounts for (a) the number of odd spheres in a building block and (b) the expectation of uneven pairs for such number of odd spheres in the building block. This differs from Eq. (3) as used in [1], where all these $\lambda_{i}$ were taken as identical in case of odd spheres in a building block.

It is beyond the scope of this article to provide a general mathematical proof of Eqs. (26) and (29) to hold for alternating regular graphs with other $n$ and $\xi$ values as examined here. But besides their validity for the bimodal fcc, bcc, and sc graphs, a brief examination showed that these equations are also valid for $n=6$, for both $\xi=3$ and $\xi=4$; see Figs. 5(a) and 5(b) for the pertaining graphs. Figure 5(a) corresponds to three triangular close-packed spheres, with on top of them the three other spheres, triangular close packed as well, each lower sphere touching one top sphere. Figure 5(b) represents the Platonic graph of the octahedron [2]. ${ }^{1}$ Hence, the analysis

\footnotetext{
${ }^{1}$ Besides the octahedron, the cube (regular hexahedron), and the tetrahedron, discussed in this paper, the two other Platonic solids are the icosahedron and the dodecahedron.
} 
of bimodal vertices and distorted edges of both structures appearing in Fig. 5 confirms that Eqs. (26) and (29) are valid for a wider range of $n$ and $\xi$ than analyzed (and needed) here.

\section{CONCLUDING REMARKS}

In this article analytical equations are derived for the bimodal packing fraction of the three crystalline cubic systems: viz. fcc, bcc, and sc. Their lattices consist of randomly placed binary hard spheres, and it is accounted for the number of distorted bonds in their building blocks, using graph theory. It appears that one general packing equation [Eq. (21)] can be derived, valid again for all 14 Bravais lattices, which are all governed by one of the three building blocks analyzed here. The three lattices and graphs studied here yield a distortion term $\lambda_{i}$ that is a quadratic function [Eq. (29)] of the number of odd spheres in the structure, in con- trast to [1] where $\lambda$ was constant (since number of distorted bonds was not accounted for). This rule appears to hold for some other planar regular graphs as well (Fig. 5).

The expressions presented in this article are thoroughly validated by comparing them with lattice data provided by [6] and by applying them to the process of amorphization. Though the present analysis is completely based on geometrical considerations, without reference to external fields, frictional contact forces, sphere compression, or entropic fluctuations, it is seen that the present basic space-filling theories on "simple" noninteracting hard spheres are a valuable tool for the study of more complicated processes and phenomena.

\section{ACKNOWLEDGMENT}

The author acknowledges the assistance of Götz Hüsken with the numerical solution of Eq. (23).
[1] H. J. H. Brouwers, Phys. Rev. E 76, 041304 (2007).

[2] J. A. Dodds, Nature (London) 256, 187 (1975); J. Dodds and H. Kuno, ibid. 266, 614 (1977).

[3] J. A. Bondy, in Handbook of Combinatorics, edited by R. L. Graham, M. Grötschel, and L. Lovász (Elsevier, Amsterdam, 1995), Vol. I; J. A. Bondy and U. S. R. Murty, Graph Theory with Applications (Macmillan Press, London, 1976).

[4] A. R. Denton and N. W. Ashcroft, Phys. Rev. A 42, 7312 (1990).

[5] P. Jalali and M. Li, Phys. Rev. B 71, 014206 (2005).

[6] W. Luck, M. Klier, and H. Wesslau, Ber. Bunsenges. Phys.
Chem. 67, 75 (1963).

[7] H. J. H. Brouwers, Phys. Rev. E 74, 031309 (2006); 74, 069901(E) (2006).

[8] W. Hume-Rothery, G. W. Mabbott, and K. M. Channel-Evans, Philos. Trans. R. Soc. London, Ser. A 233, 1 (1934); W. Hume-Rothery, R. E. Smallman, and C. W. Haworth, The Structure of Metals and Alloys, 5th revised ed. (Institute of Metals, London, 1969).

[9] T. Egami and Y. Waseda, J. Non-Cryst. Solids 64, 113 (1984). [10] S. H. Liou and C. L. Chien, Phys. Rev. B 35, 2443 (1987). 